\title{
DNA content equivalence in haploid and diploid maize leaves
}

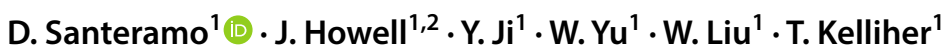

Received: 22 July 2019 / Accepted: 29 November 2019 / Published online: 9 December 2019

(c) The Author(s) 2019

\begin{abstract}
Main conclusion The qPCR assay developed to differentiate haploid and diploid maize leaf samples was unsuccessful due to DNA content difference. Haploid cells are packed more closely together with less cellular expansion.
\end{abstract}

\begin{abstract}
Increased ploidy content ( $>2 \mathrm{~N}$ ) directly correlates with increased cell size in plants, but few studies have examined cell morphology in plants with reduced ploidy (i.e., haploids). To pioneer a scalable new ploidy test, we compared DNA content and cellular morphology of haploid and diploid maize leaves. The amount of genomic DNA recovered from standardized leaf-punch samples was equivalent between these two ploidy types, while both epidermal and mesophyll cell types were smaller in haploid plants. Pavement cells had a substantially smaller size than mesophyll cells, and this effect was more pronounced in the abaxial epidermis. Interveinal distance and guard cell size were significantly reduced in haploids, but the cell percentage comprising stomata did not change. These results confirm the direct correlation between ploidy content and cell size in plants, and suggest that reduced cell expansion predominantly explains DNA content equivalence between haploid and diploid samples, confounding efforts to develop a haploid detection method using DNA content.
\end{abstract}

\section{Introduction}

In both animals and plants, the regulation of cell size is one of the most important and well-studied aspects of development. In plants, endo-reduplication is a developmentally regulated phenomenon, and cell size is roughly proportional to ploidy content (Roeder et al. 2012). This has been well-studied in tissues and cell types with increased $(>2 \mathrm{~N})$ ploidy (Sugimoto-Shirasu and Roberts 2003). Still, there is room for further advancement in the discoveries that have already been made that relate to haploid $(1 \mathrm{~N})$ maize plants. Prior studies have investigated stomata length with respect to ploidy determination and doubled haploid (DH) production systems. Stomata guard cell length may be used to differentiate true haploid and doubled haploid plants from false positive and diploid plants as early as the Leaf 2 stage in maize (Choe et al. 2012). However, even in more recent studies

D. Santeramo and J. Howell contributed equally to this work.

D. Santeramo

dylan.santeramo@syngenta.com

1 Seeds Research, Syngenta Crop Protection, LLC, Research Triangle Park, Durham, NC, USA

2 Pairwise Plants, Research Triangle Park, Durham, NC, USA investigating stomata lengths, there are issues in application of this method for large-scale DH production systems due to the large variation in stomata length between haploid and doubled haploid plants, in addition to the small difference in means between these groups (Molenaar et al. 2019).

Haploid plants are used to rapidly generate inbred lines to accelerate breeding: one generation of haploids can save seven generations compared to single-seed descent (Chang and Coe 2009). Haploid sporophytes are short, male-sterile, and have thinner leaves compared to their diploid counterparts. In certain crops, haploid plants may undergo somatic genome duplication; in lieu of this, colchicine treatment may be used to induce genome doubling and male fertility, enabling self-pollination and the production of homozygous (doubled haploid, or DH) progeny seed: instant inbred lines. Haploid induction and DH breeding pipelines are under development in academic and commercial settings in agriculturally significant crops, either through knock-out of homologs of the MATRILINEAL gene (Yao et al. 2018) or through alteration of the CENTROMERIC HISTONE3 (CENH3) gene (Ravi and Chan 2010). In both of these systems, the mutant haploid induction phenotype exhibits incomplete penetrance, and is accompanied by significant levels of diploid hybrids (normal seed) and aborted embryos likely due to endosperm failure. In the maize matl haploid 
induction mutant for instance, between 3 and $15 \%$ of viable progeny are haploids, while the rest are diploid hybrids (Kelliher et al. 2017).

Haploids may be identified and sorted away from their diploid hybrid counter parts via presence or absence of a dominant genetic or phenotypic marker present in the haploid inducer line. In maize the R1 dominant inducer of anthocyanin accumulation is used. This gene is typically homozygous in the matl haploid inducer lines that are used as male parents in the haploid induction crosses. The R1 marker can be recognized in mature or immature seed based on anthocyanin accumulation and purple color expression in the scutellum, a transfer tissue surrounding the embryo (Nanda and Chase 1966): diploid hybrids develop this color and are discarded; maternal haploids lack the paternally derived R1 marker gene and remain cream-colored (Chaikam et al. 2015).

Newly developed DH pipelines in other crops would require an analogous visual identification system, but if an obvious candidate gene cannot be used (Yao et al. 2018), an alternative method of haploid plant identification would be required. Ploidy detection via flow cytometry is one option to quickly test the success of chromosome doubling treatments by distinguishing which haploid plant sectors have undergone endo-reduplication (or genome doubling): however, this method has historically been constrained by high cost and low throughput.

Recent improvements in ploidy analysis equipment and software have enabled automated processing of 96-well plates (Suni et al. 2003), and crushing devices for nuclei extraction can be used to speed processing time (MEKU Erich Pollähne 2019). However, for haploid identification at industrial scale ( $>1,000,000$ haploid per year), flow cytometry is still not a feasible approach. We hypothesized that one may distinguish doubled haploid cell lineages and plants from haploids using a qPCR reading of DNA content from a standard-sized leaf sample. One technician can process 7000 leaf samples, including both DNA extraction and qPCR setup, in approximately 5-6 h; the full experiment, including analysis, can be accomplished in $24 \mathrm{~h}$.

The current approach for relative copy number analysis by duplex TaqMan assays is not suitable for ploidy analysis, since both targeted genes will most likely be in the same ratio in haploids and diploids, regardless of total DNA amount in each cell. Utilizing methods such as a Qubit dsDNA assay or PicoGreen would not work because they are designed for pure DNA, while the samples discussed in this study are cell lysate. PicoGreen can bind to and be used to measure dsDNA, ssDNA, RNA, and protein as long as they are relatively pure and homogeneous in size. Several commercial kits are available for specific applications. However, the PicoGreen methods rely heavily on standard controls which must be equivalent to unknown samples, in terms of both purity and size, and can differ significantly as a result of damage to the materials and methods for their preparation. Additionally, plant leaf tissues have plenty of metabolites and fluorophores, whose green appearance interfere with the PicoGreen method if not removed.

Qubit dsDNA assays are routine for purified plasmid and genomic DNA where the DNA is fairly pure and fragment sizes are comparable to the DNA standard. When this is not the case, it can cause two- to $>$ tenfold overestimate or underestimate of DNA quantity. On the other hand, qPCR analysis paired with relevant TaqMan assays are a more specific and accurate method for DNA quantification for crude DNA in plant leaf cell lysate. Prior studies have reached similar conclusions for FFPE-DNA (Nakayama et al. 2016). However, a singleplex TaqMan assay, constituting a PCR run with a single TaqMan assay in replicates, could perhaps be used to consistently distinguish differences in DNA content, assuming that haploid samples have a significant enough reduction in DNA content compared to diploid samples.

If this idea worked, one could imagine a $\mathrm{DH}$ production scheme in which leaf sample qPCR distinguished haploid from diploid young seedlings. Following haploid colchicine treatment, a second leaf punch sample could be used to establish the success of chromosome doubling; doubled plants could then be transplanted and self-pollinated in the field.

To test this idea, we quantified the DNA extracted from equal sized leaf punches from haploid and diploid plants from the same genetic background. While haploid leaf samples overall had slightly less DNA, the qPCR analysis showed no significant difference between individual haploid and diploid leaf punches. To ask whether this may be due to denser packing of haploid cells, we looked at the relationship between ploidy content and cell size in equivalent haploid and diploid leaf punches, focusing on the epidermis and mesophyll. The broad view of our findings are consistent with prior studies and explain why we find that reduced haploid cell expansion reveals why roughly the same amount of DNA is captured from leaf punches of haploid and diploid plants.

\section{Materials and methods}

\section{Isolation of haploid and diploid NP2222 embryos and subsequent germination of the plants}

NP2222 inbred and haploid inducer lines were grown in the Syngenta Crop Protection Advanced Crop Lab in Research Triangle Park, NC under standard irrigation and fertilization regimes to maintain robust growth. The greenhouse was maintained at $22-30{ }^{\circ} \mathrm{C}$; supplemental lighting was provided from 06:00 to 20:00 daily. 
Leaf disk samples were used in this experiment for both DNA and microscopic analysis. These samples were taken from ten haploid and ten diploid maize plants of the same genetic background to eliminate cell or tissue patterning differences due to genetic variation. The samples were obtained from plants germinated from isolated embryos, which were harvested from ears produced by a self-pollination of NP2222 (diploids) or a haploid induction cross of NP2222 by the male haploid-inducing line 09BD900336, which is homozygous for the native matl-1 mutant allele and the R1 gene (Kelliher et al. 2017). The embryos were extracted from one diploid ear and two haploid induced ears harvested at 17 days after pollination (DAP).

Maternal haploid embryos carrying only the NP2222 genome were identified $24 \mathrm{~h}$ after isolation by the absence of purple color (as they lacked the R1 gene); these haploids were separated from purple, diploid hybrid embryos. In contrast, the diploid NP2222 embryos were generated by self-pollinating NP2222. These embryos were the same size, shape, and appearance of the haploids. Both sets of embryos were germinated and grown for 14 days in three separate phytatrays (one for each ear). The phytatray media contained sucrose-supplemented minimal medium with a gelling agent, and grew in a growth chamber with $16 / 8$ day/ night conditions and ambient temperature $\left(\sim 25^{\circ} \mathrm{C}\right)$. Light intensity between 50 and 400 PAR may be used to germinate and grow embryos within the growth chamber. After 14 days of growth under in vitro conditions, the seedlings were potted in soil in the greenhouse. After 14 days of growth in the greenhouse, samples were taken for both DNA and microscopic analysis (see below for processing of the samples).

\section{DNA preservation and extraction from leaf tissue}

To minimize variations in DNA content extracted from leaf tissues of the ten diploid and ten haploid plants, the experiments were conducted side-by-side.

All samples were collected at the same time of the same day.

For the leaf disk samples collected for DNA analysis, as well as those that were later studied by microscopy, four leaf punches, each $6 \mathrm{~mm}$ in diameter, were sampled from the third emerged leaf with a visible leaf collar (V3 leaf) (Abendroth et al. 2011) of each plant. The samples to be later used for PCR were loaded into the same 96-well sample block on ice. Those to be used for microscopy were sampled and placed into vials on ice, to which the clearing solution was later added.

The idea and theory behind this study are represented by Fig. 1a. If the relationship between ploidy level, cell size, and DNA content between diploids and haploids were $2: 1$, then we should observe approximately twice the amount of DNA content in diploid cells when compared to haploid cells (Fig. 1a). Plants were sampled in such a way that each punch included a portion of the midrib of the V3 leaf, so that the midrib could provide a visual aid as to which surface of the leaf was abaxial and adaxial when staged on a slide for confocal microscopy. One might assume that diploid plants would develop at a more advanced rate compared to haploid plants, however, this difference is not as pronounced in the early vegetative growth stages, such as at the V3 stage (Abendroth et al. 2011). Figure $1 \mathrm{~b}$ is an example diagram of a cross section of maize leaf tissue. The colors in the associated legend represent the various tissues within a leaf, with the largest vascular bundle in the diagram representing the midrib.

To prevent DNA damage and yield loss during sample preparation, we pioneered an integrated, simple method for preserving and extracting precise amounts DNA from leaf tissues. This method is based on a new combination of chemicals covering multiple critical aspects of the DNA preparation, including DNA protection, cell lysis, DNA release and recovery, and control of PCR inhibitors derived from the leaf tissue. Briefly, the new method mainly considered (1) preservation of leaf DNA from damage after leaf collection by the chemical combination of glycerol known as cryoprotectant plus antioxidant polyvinylpyrrolidone plus EDTA in high $\mathrm{pH}$ solution, (2) raw DNA extraction for leaf tissue with high speed grinding in alkaline solution so to enhance cell lysis and DNA release, (3) raw DNA in this cell lysate with carefully optimized chemical combination for eliminating major inhibitors to direct qPCR analysis, without any further cleanup which affect total DNA recovery and introduce more variations between samples. More details about this method can be found in the patent publication (Ji 2018). The preservation and extraction (PE) mixture consists of $20 \mathrm{mM} \mathrm{NaOH}$, $15 \%$ (w/v) glycerol, $0.5 \%$ polyvinylpyrrolidone (PVP)40 (Sigma-Aldrich), and $2 \mathrm{mM}$ EDTA. The raw DNA extracted from leaf tissue is ready for qPCR analysis and is stable for at least weeks at ambient temperatures.

The procedure from leaf sampling to PCR-ready DNA is as follows:

- Collect 4 full leaf disks per plant and load 1 disk per well in sample block on ice.

- Dispense $250 \mu \mathrm{l}$ of PE into each well containing leaf tissue. The tissue can be kept in PE in a closed sample block at ambient temperature (RT) for weeks, optionally.

- Dispense one 3/16" steel bead per well and seal the block. *After this step, the DNA extraction can be done at any time within 1 week.

- Grind leaf tissues in the block for 3 min using a GenoGrinder at $1600 \mathrm{rpm}$.

- Centrifuge the block for $15 \mathrm{~min}$ at $4000 \mathrm{rpm}$ at room temperature. 

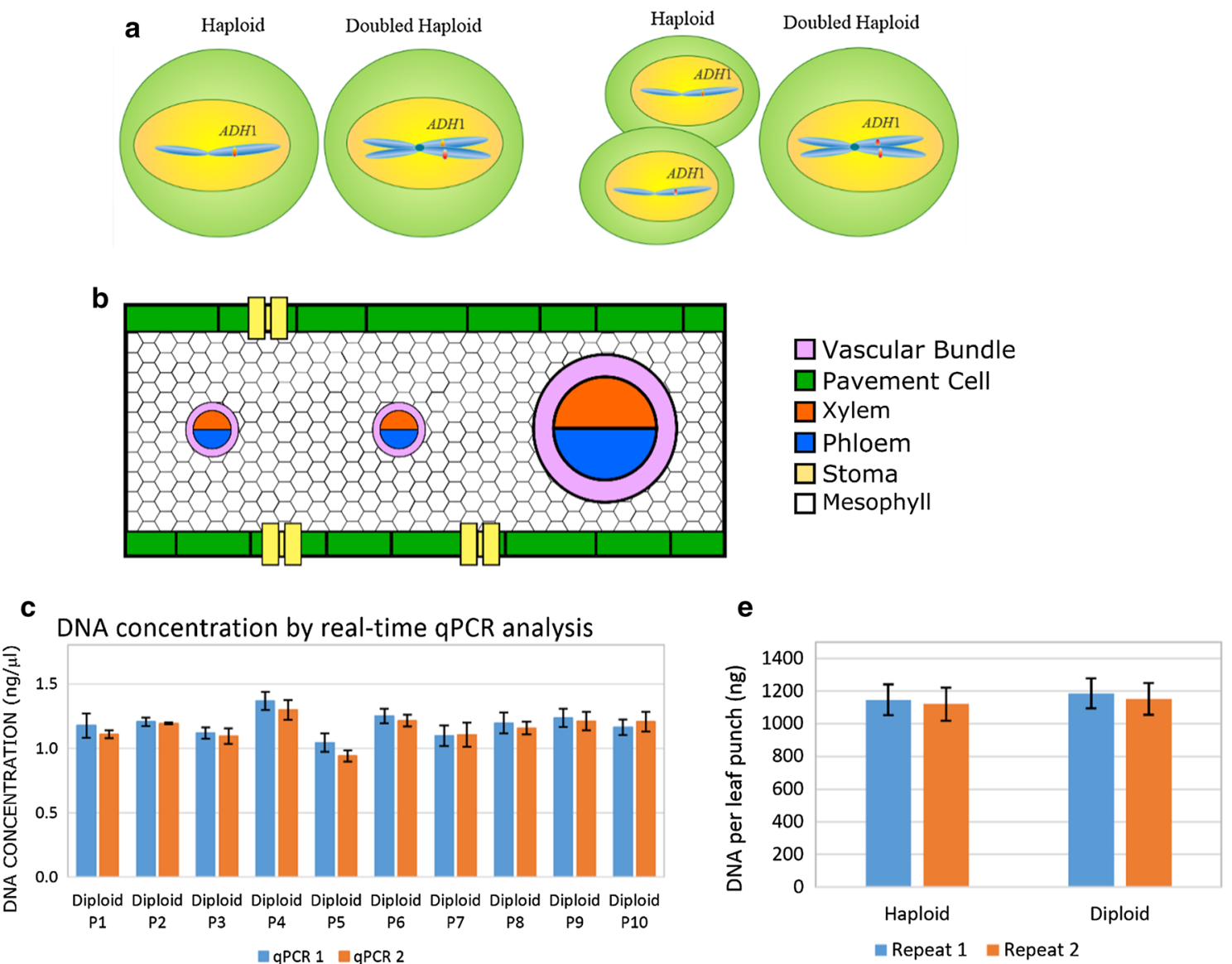

\section{DNA concentration by real-time QPCR in haploid and diploid leaf punches}

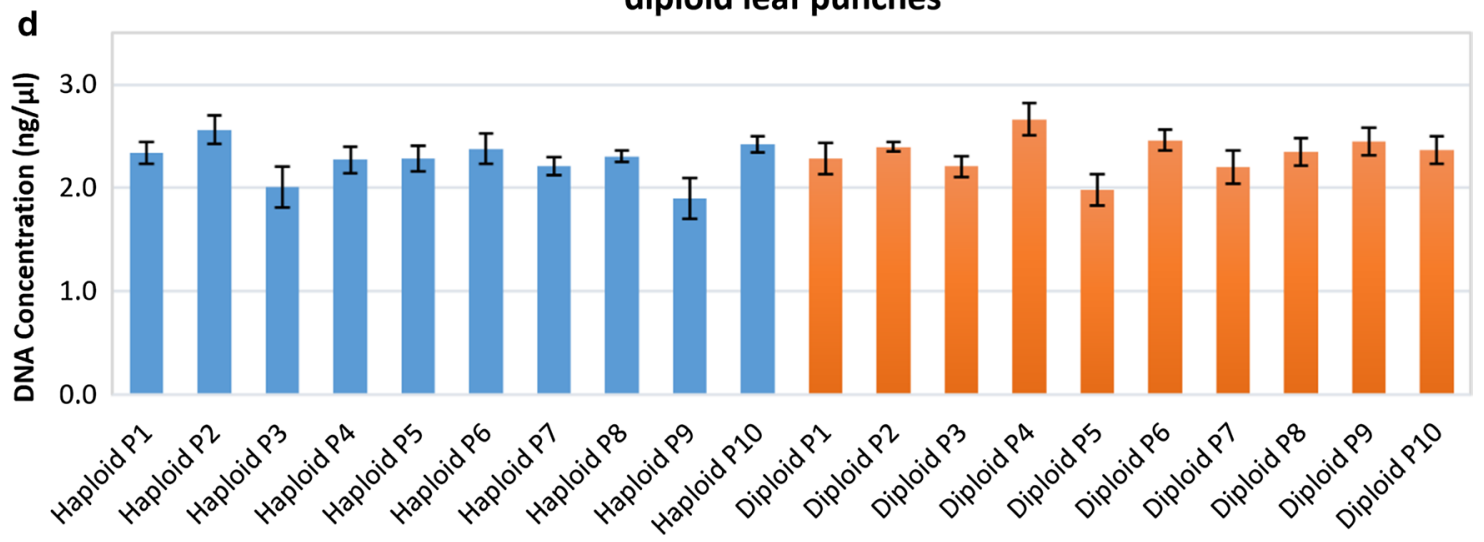

- Transfer $100 \mu \mathrm{l}$ of supernatant per well into a new plate, and mix with $100 \mu \mathrm{l}$ of $20 \mathrm{mM}$ Tris- $\mathrm{HCl}$ buffer, $\mathrm{pH}$ 7.5. The DNA is ready for qPCR analysis and storage.

*To ensure maximum efficiency of cell lysis, four additional, smaller steel beads (1/16") may be added, and the grinding step may be repeated, after $1 \mathrm{~min}$ of centrifugation.

\section{Real-time PCR for quantitative DNA measurement}

Real-time PCR via TaqMan assay for quantitative DNA analysis is the most sensitive and accurate method for a wide variety of applications (Deepak et al. 2007). The specificity of the TaqMan assays targeting an endogenous maize gene (e.g., maize ALCOHOL DEHYDROGENASE1 [ZmADH1]) is critical for this study, as we wanted to determine the amount of DNA in cell lysates relative to a standard DNA control. The qPCR method we used has been validated by 
4 Fig. 1 a Two scenarios showing the relationships between quantity of gDNA (measured with qPCR for $A D H 1$ gene) and ploidy of maize. (Left) If the cell size in both haploid and doubled haploid maize leaves are the same, then the quantity of gDNA in doubled haploid will be twice that of those in the haploid plants (i.e., the $C \mathrm{t}$ value of $A D H 1$ genes in the doubled haploid plants will be one $C t$ lower than those from the haploids). (Right) However, in the situation we actually observed in this study, the cell size of haploid plant is approximately a half of those from the doubled haploid plants, so the total amount of gDNA in the same area (e.g., one punch of leaf disc) will be similar between the haploid and doubled haploid plants (with no obvious $C t$ value difference in qPCR). b Diagram of a cross section of a maize leaf with corresponding legend for tissue types. c DNA concentration analyses between haploid and diploid plant leaf tissue samples a. The DNA concentrations as determined by two replications of real-time qPCR for the $A D H 1$ gene from ten diploid NP2222 plants. Each bar is an average of the DNA concentration as measured via $\mathrm{qPCR}$ from four distinct DNA preparations from four individual leaf punches. d DNA concentration as measured by qPCR from four leaf disks sampled from each of ten haploid and ten diploid plants. The DNA yields were represented by DNA concentrations. The results were very similar both within and between the haploid and diploid groups. The error bars in Fig. 1c, $d$ are standard deviations of DNA samples from four individual leaf punches per plant (technical replicates). e Total DNA amount per leaf punch from haploid and diploid plants. Results from the two qPCR repeats were similar and the average DNA amount per leaf disk was nearly the same. The error bars in Fig. 1e are standard deviations from ten plants per group (biological replicates)

the Joint Research Centre of the European Commission for the detection of purified maize DNA (Larcher et al. 2008), where this gene $(A D H 1)$ is used as an endogenous control in standard duplex qPCRs.

$A D H 1$ is a single copy gene and broadly used as an endogenous control in relative copy number analysis for a target gene by real-time PCR (Scholdberg et al. 2009). Since other genes will be maintained in the same ratio as $A D H 1$ in either haploid or diploid samples (in theory), we only need to use the single real-time PCR assay targeting the endogenous gene $A D H 1$ to determine the DNA concentration within the cell lysate, with a certified maize DNA as standard control (value not shown). The reactions used in nearly all samples were standardized and validated by the JRC (Larcher et al. 2008), with the exception of the cell lysate used in Fig. 1c. For this, a twofold dilution was used to avoid any inhibition to the qPCR for accurate DNA quantification. The probe used in this qPCR is the same as the one employed in the JRC report (Larcher et al. 2008), with the sequence as follows:

\section{Zm adh1-P Probe: VIC 5'-AAT CAG GGC TCA TTT TCT CGC TCC TCA-3' TAMRA}

The procedure for qPCR analysis was slightly modified for this study. In the PCR set-up, $5 \mu \mathrm{l}$ of supplemented Sigma Jumpstart ReadyMix $(\times 2), 0.3 \mu$ l of each primer $(10 \mu \mathrm{M})$, $0.2 \mu \mathrm{l}$ of probe $(10 \mu \mathrm{M}), 1.2 \mu \mathrm{l}$ nuclease free water, and
$3 \mu \mathrm{l}$ of DNA are used. The cycling parameters were $95^{\circ} \mathrm{C}$ for $5 \mathrm{~min}$, followed by 40 cycles of $95{ }^{\circ} \mathrm{C}$ for $10 \mathrm{~s}$ and $60{ }^{\circ} \mathrm{C}$ for $30 \mathrm{~s}$. The reference maize DNA sample (Larcher et al. 2008) served as a standard to estimate absolute DNA concentration.

\section{Plant sample processing for confocal and light microscopy}

Ten haploid and ten diploid plants were sampled at the same time of day at exactly 14 days after potting using a handheld leaf puncher, just as described above for sampling for qPCR. The following fixing and staining protocol was developed:

\section{Day 1: sample fixation and dehydration}

Two leaf disk punches were taken from each plant from the middlemost region of the fully emerged third leaf of the V3 stage maize plant, and loaded into $1.5 \mathrm{ml}$ microcentrifuge tubes. The samples included a portion of the leaf midrib which aided with orientation during the eventual mounting and imaging on the microscope slide. Leaf disk samples were fixed in $1 \mathrm{ml}$ of $4 \%$ FAA + PBS on ice in a vacuum desiccator (Space Saver, Bel-Art SP Scienceware) set to 400 millibars of pressure for $2 \mathrm{~h}$ (modified from Kurihara et al. 2015). Samples were washed in $\times 1$ PBS for 1 min on a platform shaker (Innova 2000, New Brunswick Scientific) at $145 \mathrm{rpm}$, followed by $1 \mathrm{~min}$ under vacuum. To aid in tissue clearing, the samples were dehydrated in five increasing $(70 \% \times 2,90 \%$, and $100 \% \times 2)$ concentrations of ethanol for $30 \mathrm{~min}$ each, followed by an overnight incubation in $100 \%$ ethanol. These dehydration steps were performed on a shaker set at $145 \mathrm{rpm}$.

\section{Day 2: leaf disk samples cleared}

"ClearSee" solution (10\% xylitol, $15 \%$ sodium deoxycholate, and 25\% urea (Kurihara et al. 2015) replaced the PBS, and the tissue samples were then incubated for $2 \mathrm{~h}$ under the 400 mbar vacuum pressure, and then left to clear at room temperature for 5 days on the shaker at $145 \mathrm{rpm}$.

\section{Day 7: leaf disk samples stained and rinsed}

After 5 days, the transparent samples were stained with a $1 \mu \mathrm{g} / \mathrm{ml}$ Calcofluor White in ClearSee solution for $30 \mathrm{~min}$ under vacuum infiltration and $30 \mathrm{~min}$ on the shaker. The staining solution was removed, and ClearSee was added to help clear away any residual stain. The leaf disk samples remained rinsing in ClearSee for two additional weeks, on the shaker set at $145 \mathrm{rpm}$. 


\section{Day 21: confocal imaging of leaf disk samples}

Before imaging on the confocal microscope (LSM 710, Zeiss), the samples underwent one final rinse in ClearSee under vacuum for $30 \mathrm{~min}$, followed by $30 \mathrm{~min}$ on the shaker. The midrib on each leaf disk sample was removed, prior to mounting on the microscope slide, and used as a reference to mount the abaxial side of the leaf disk facing up. This ensured consistent mounting orientation of all samples. ClearSee was also used as a mounting medium. A cover slip was then placed on top of the sample on the slide, with mounting clay applied to the four corners. Images were captured using a $20 \times$ objective, $202 \mu \mathrm{m} \times 202 \mu \mathrm{m}$ field of view, Speed 9, Interval 1, bi-directional scanning, and a $1024 \times 1024$ frame size. Three Z-Stacks images were taken per leaf disk sample from three different locations on the leaf disk. To avoid including any portion of the leaf disk twice, the joystick on the confocal microscope was moved three times, to shift the field of view in a linear fashion, to the next new field of view that would be imaged. Corrections were used throughout each Z-Stack, to aid in visualizing cells that were in deeper leaf tissue layers. This confocal imaging process generated three Z-Stacks per leaf disk, for a final total of 60 haploid Z-Stacks and 60 Diploid Z-stacks generated from a population of ten haploid and ten diploid plants.

\section{Analysis}

To compare cellular morphologies between haploid and diploid leaf disks, we fixed, cleared (Kurihara et al. 2015), stained, and collected images of the 40 leaf disks, capturing three images from each of the 20 haploid and 20 diploid leaf disks for a total of 120 images. Leaf disks were sampled at the same time of the day and from the same position on the V3 leaf of each plant. Images were captured from the same position and orientation with respect to the leaf's major vein. On the abaxial surface of the leaf, cell counts and $X$ and $Y$ dimension measurements were made of pavement cells within the $202 \mu \mathrm{m}$ square in the image window, and of mesophyll cells within a $200 \mu \mathrm{m} \times 100 \mu \mathrm{m}$ field of view located directly under a minor leaf vein.

ZEN Black software (Carl Zeiss) was used to count and measure cells within leaf disks. The line and scale bar tools were used to aid in counting and measuring cell lengths and widths. Using the $10 \times$ transmitted light objective on a Ts2R microscope (Nikon Eclipse), in conjunction with NISElements Basic Research software, additional images and data were collected on all 40 of the haploid and diploid leaf disk samples. Within-frame stomata counts and interveinal widths were measured, and pavement and stomata cell patterning was modeled in Microsoft Excel. This was done by translating the positioning and quantity of pavement cells and stomata to corresponding cells within Excel, so as to represent the lengths and counts of the cells within visual frame as observed in Fig. 4d, e.

\section{Results}

As shown in Fig. 1c, the DNA preservation and extraction method gave highly repeatable qPCR results and led to a consistent reading of DNA quantification from two replicates of the same plants. A dilution series also confirmed that DNA quality is suitable for quantification by qPCR analysis (results not shown). Given such a sensitive and specific TaqMan assay for $A D H 1$, the DNA quantifications can be easily replicated. The average relative standard deviation (RSD) was about 5\%. The DNA amounts (represented in concentrations) from 4 individual leaf punches per plant were consistent, with RSDs from 1 to $9 \%$. The biggest difference of DNA amounts in these diploid plants was about 33\% (P4 vs. P5). However, the DNA amounts in the rest of eight plants were very similar $(1.17 \pm 0.05 \mathrm{ng} / \mu \mathrm{l})$ and the overall average DNA amount was also $1.17 \mathrm{ng} / \mu \mathrm{l}$.

Employing this method to compare haploid and diploid leaf punch DNA quantity, we sampled ten genetically identical, NP2222-inbred haploid and diploid plants (see "Materials and methods"). We sampled four leaf punches from the mid-central V3 leaf of each plant exactly 14 days after potting to soil, which occurred after the 14 days germination period in vitro. The average DNA content per leaf disk was consistent between replicates and similar between haploid and diploid plants (Fig. 1d). The difference between qPCR repeats $(97.6 \% \pm 5.3 \%)$ was within the range of typical mechanical errors among other variations. The difference between haploid and diploid plants on average $(97.0 \% \pm 9.5 \%)$ was almost negligible. All calculations were based on individual and full leaf disk without prior knowledge of total number of cells included.

With qPCR analysis, we were able to see some differences between the sets of haploid and diploid plants (Fig. 1e), but if the highest and lowest DNA amounts from each group are removed as outliers, the average DNA concentration per leaf punch of the remaining eight plants was nearly identical, $2.28 \pm 0.13 \mathrm{ng} / \mu \mathrm{l}$ for haploids vs. $2.34 \pm 0.10 \mathrm{ng} / \mu \mathrm{l}$ for diploids. Since diploid cells carry twice the amount of chromosomal DNA than haploid cells, these results support the inference that haploid plants pack the cells in leaf tissues differently with respect to cell size (cells per leaf area).

To ensure that the embryos used in this study were truly haploids and not false positives (diploid embryos not expressing scutellum color change), the germplasm used in this study has been thoroughly tested and has $\mathrm{a}<0.1 \%$ false positive haploid identification rate using the R1 color marker. Additionally, all 20 haploid leaf disk 
samples studied derived from the 10 haploid plants did have smaller cell sized based on morphological analyses.
The abaxial and adaxial pavement cells were visibly smaller in the haploid (Fig. 2a, c) compared to the diploid cells (Fig. 2b, 2d). A statistically significant difference
Fig. 2 Representative images of abaxial and adaxial epidermises within a $202 \mu \mathrm{m} \times 202 \mu \mathrm{m}$ field of view. Gamma was adjusted on all images to aid in visualization. a Haploid and $\mathbf{b}$ diploid abaxial epidermal cells. c Haploid and d diploid adaxial epidermal cells. e, f Abaxial epidermis average cell totals (e) and cell areas $\mathbf{f}$ from the 120 leaf disk images (comparing 60 haploid to 60 diploid images). $\mathbf{g}$, h Abaxial and adaxial average epidermal cell counts (g) and average epidermal cell area (h) calculated from five haploid and five diploid Z-Stack images were selected for additional analysis based on the clarity of the adaxial epidermis. The data was compared using a Student's $t$ test and statistical values shown as: ns (not significant), $P>0.05, * P \leq 0.05, * * P \leq 0.01$, $* * * P \leq 0.001$, **** $P \leq 0.0001$.

Standard error bars and percent difference calculations were added to each graph. $50 \mu \mathrm{m}$ scale bars added to each image
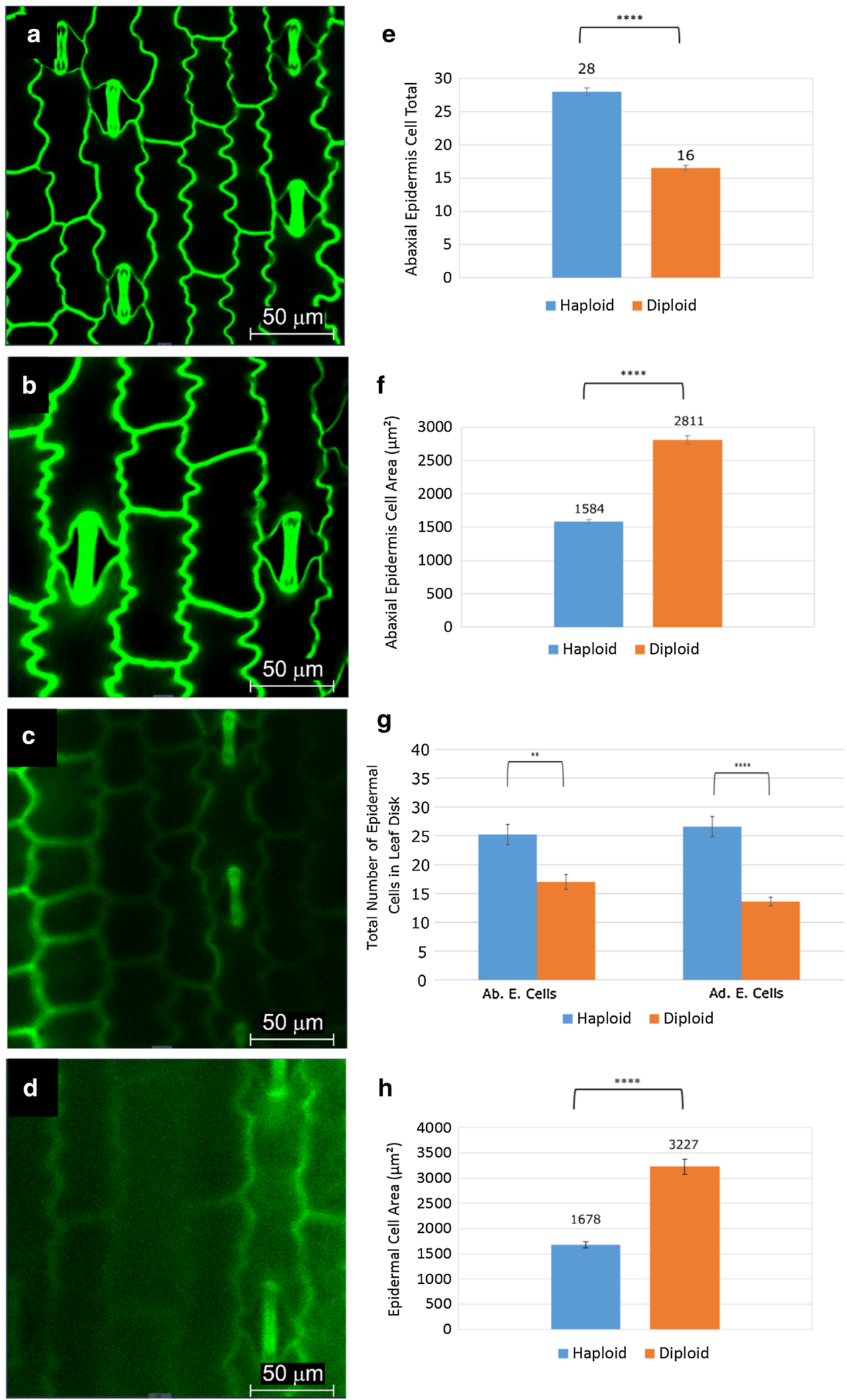

h
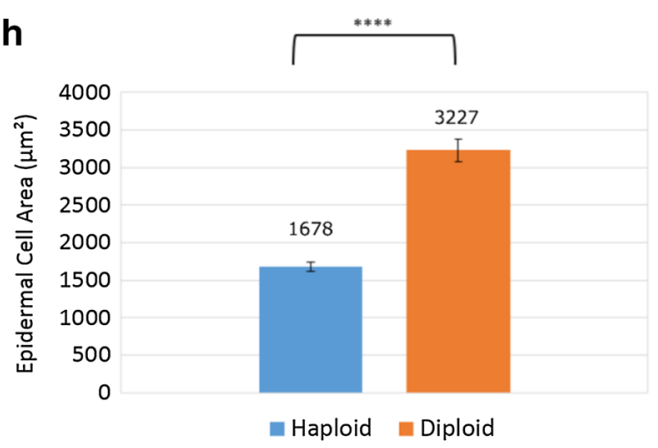
between haploid and diploid samples was observed for abaxial epidermal pavement cell count and the amount of leaf surface area covered per cell. The haploid leaf disks had $69.5 \%$ more abaxial epidermal pavement cells (Fig. 2e). Each haploid cell comprised $19.6 \%$ less leaf surface area than their diploid counterparts (Fig. 2f). The difference was more extreme on the adaxial side of the leaf: there were $95.6 \%$ more adaxial epidermal pavement cells per unit area in haploid versus diploid leaf disks (Fig. 2g). Combined together, the average area occupied by a single pavement cell was $48 \%$ lower in haploids (Fig. 2h).

There was no significant difference observed between the cell counts or cell sizes of the abaxial mesophyll cells that are found within the $200 \mu \mathrm{m} \times 100 \mu \mathrm{m}$ region lying just beneath the minor leaf veins (Fig. 3a, b, e). However, to obtain a more comprehensive understanding of the mesophyll cell content of the leaves, those mesophyll cells that were closer to the adaxial surface, as well as lying beyond either side of the minor leaf vein (Fig. 3c, d) were incorporated into the new calculations. Subsequently, further investigation uncovered a difference in haploid and diploid mesophyll cell sizes and counts in the interveinal space. Haploid mesophyll cells in the interveinal space were $33 \%$ smaller (in terms of $X-Y$ area), and $34 \%$ more numerous, than their diploid counterparts. Taken together, there were $21.9 \%$ more mesophyll cells in haploid leaf disks (Fig. 3f), but this difference is largely due to fact that the interveinal mesophyll cells were significantly larger (and therefore less numerous) in diploids (Fig. 3f, g).

To ask whether the interveinal distance and overall stomata cell patterning was similar in the haploid and diploid leaf disks, a light microscope was utilized to reexamine one leaf disk from each pair of samples taken in the initial collection phase. Figure $4 a, b$ are representative images of the venation within haploid and diploid leaf tissue, respectively. The measurements and lines on each image highlight the interveinal distance within the leaves. Data were collected from three different positions on each of the leaf disks, again examining the abaxial layer. The diploid leaves had $19.6 \%$ more interveinal distance between the minor leaf veins (Fig. 4c). Diagrams were created to clarify the placement and frequency of pavement cells and stomata in the abaxial and adaxial epidermis (Fig. 4d, e). In these diagrams, the letter "E" represents a pavement cell and "S" represents a stoma. In the 20 samples examined, we found that haploid leaf disks had $65.3 \%$ more stomata per disk (Fig. 4f). Despite haploids having more numerous stomatal cells than diploids per unit of leaf area, there was no significant difference in the overall percent cell composition in total between the haploid and diploid leaf disks (Fig. 4g).

\section{Discussion}

This study examined whether a real-time quantitative PCR program could distinguish haploid and diploid plants. This type of assay would be useful as a haploid detection method or as an alternative to ploidy analysis, to clarify whether certain plant sectors were haploid or doubled haploid. This method could help determine the success of chromosome doubling treatments, by indicating which sectors are likely to give rise to flowers that can successfully produce viable gametes. Using a new DNA extraction method, we isolated leaf DNA suitable for accurate DNA quantification by real-time qPCR. We found that there was virtually no difference in the quantity of DNA obtained from leaf disks sampled from V3 leaves of haploid and diploid plants. An investigation of cell sizes of pavement and mesophyll cells from the leaf punches indicated that the reason for this equivalence was that haploid plant cells are smaller than those of diploids. Combined together, these factors offset the absence of a second genome (homologous partner) and explain how plants of different ploidy may have roughly equivalent amounts of DNA per unit leaf area.

Having suitable DNA quality and quantity as input for the real-time PCR (qPCR) analyses is critical. Since the dynamic range of input DNA in qPCR is fairly large, from a few picograms to hundreds of nanograms per reaction, a few nanograms of DNA used in this study is appropriate. DNA quality is also critical. Relative copy number analysis using duplex qPCR for a target gene and an internal endogenous control gene in the same reaction is not suitable for distinguishing haploid and diploid plants. To determine the DNA amounts obtained from leaf samples from either haploid or diploid plants, we showed that a single qPCR assay for an endogenous control gene can be used. Since variations in sample preparations can be potentially larger than any difference of DNA amounts in between individual plants, we verified the results by repeated qPCR analyses.

To determine the DNA quantity in leaf tissues reliably, this study required minimizing all major variations potentially introduced from the sampling or DNA preparation. We tried to achieve optimal DNA recovery from a single leaf punch and eliminated major variations with the following approaches: (1) complete leaf tissue grinding with different metal bead combinations, (2) efficient nucleic acid release in high $\mathrm{pH}$ reagents and prevention of DNA damage with the chemical combination, (3) minimization of further DNA manipulation (purification/precipitation) to avoid loss of DNA and large variation between samples (Xin and Chen 2012). This minimization is a result of using DNA in cell lysate, which is suitable for direct 
Fig. 3 Representative images of abaxial and adaxial mesophyll cells within a $202 \mu \mathrm{m} \times 202 \mu \mathrm{m}$ field of view. Gamma was adjusted on all images to aid in visualization. a Haploid and $\mathbf{b}$ diploid abaxial mesophyll cells. c Haploid and d diploid adaxial mesophyll cells. e Abaxial mesophyll average cell counts calculated from the entire 120 image data set. f Combined abaxial and adaxial mesophyll cell counts for cells outside of the $200 \mu \mathrm{m} \times 100 \mu \mathrm{m}$ region below a minor leaf vein, and mesophyll combined totals for both inside and outside the $200 \mu \mathrm{m} \times 100 \mu \mathrm{m}$ region below a minor leaf vein. $g$ Average combined mesophyll cell area. The graphs in $\mathrm{f}$ and $\mathrm{g}$ were calculated from five clearest haploid and diploid Z-Stack images selected for additional analysis. The data was compared using a Student's $t$ test and statistical values shown as: ns (not significant), $P>0.05, * P \leq 0.05$, $* * P \leq 0.01, * * * P \leq 0.001$, $* * * * P \leq 0.0001$. Standard error bars and percent difference calculations were added to each graph. $50 \mu \mathrm{m}$ scale bars added to each image
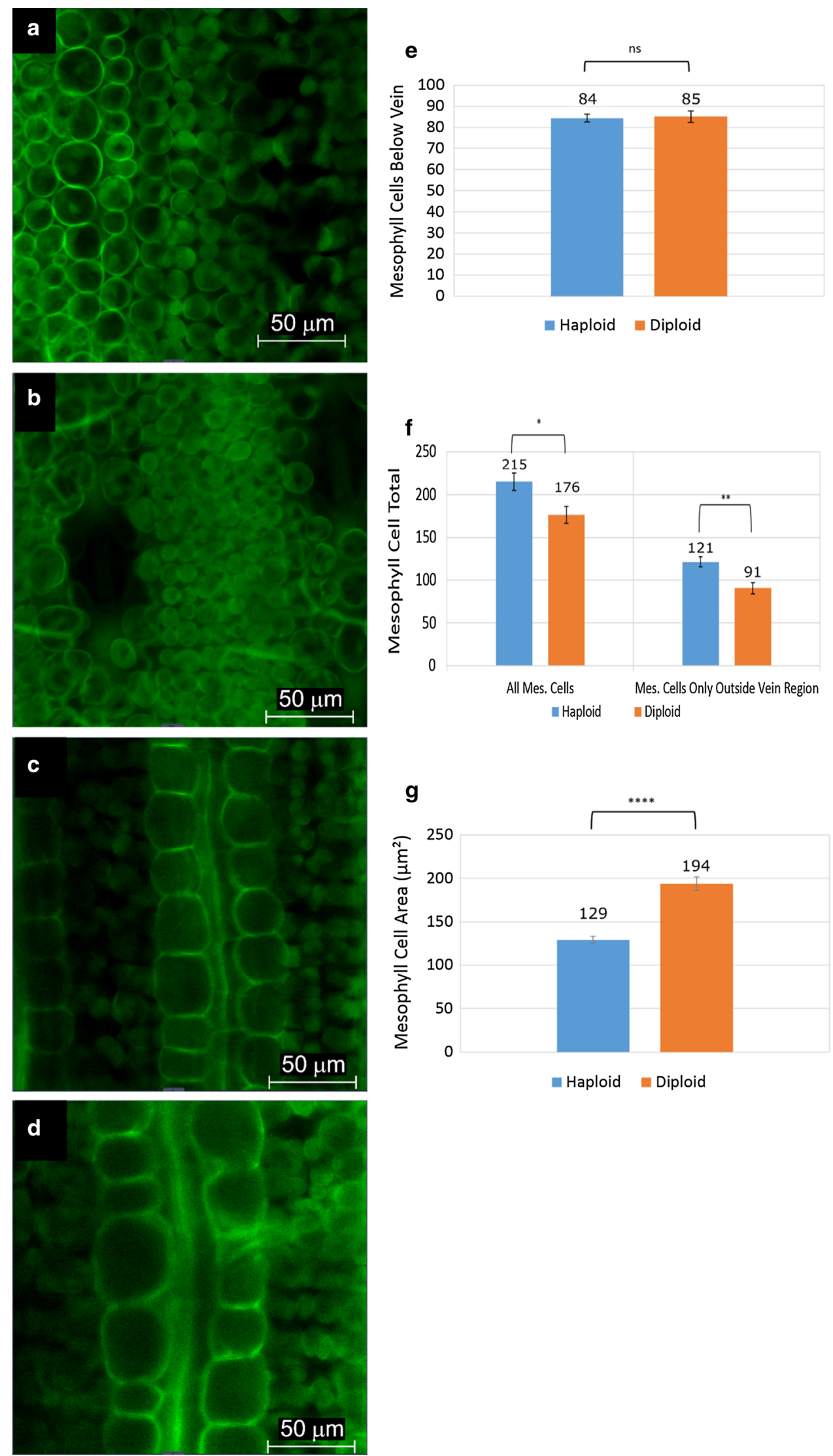
a

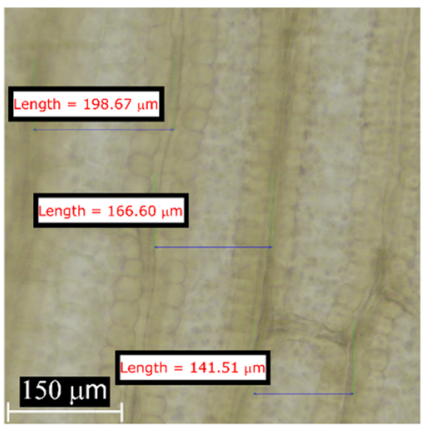

b

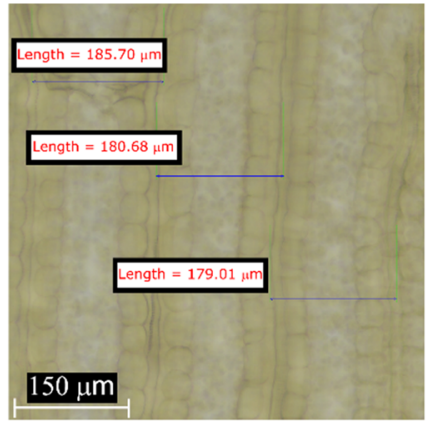

C

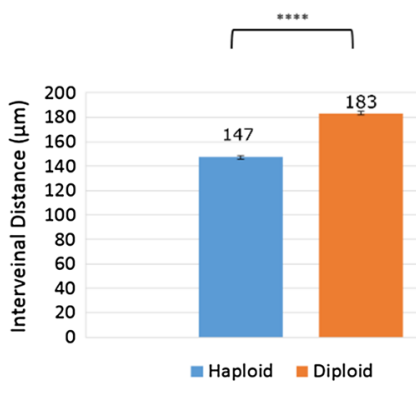

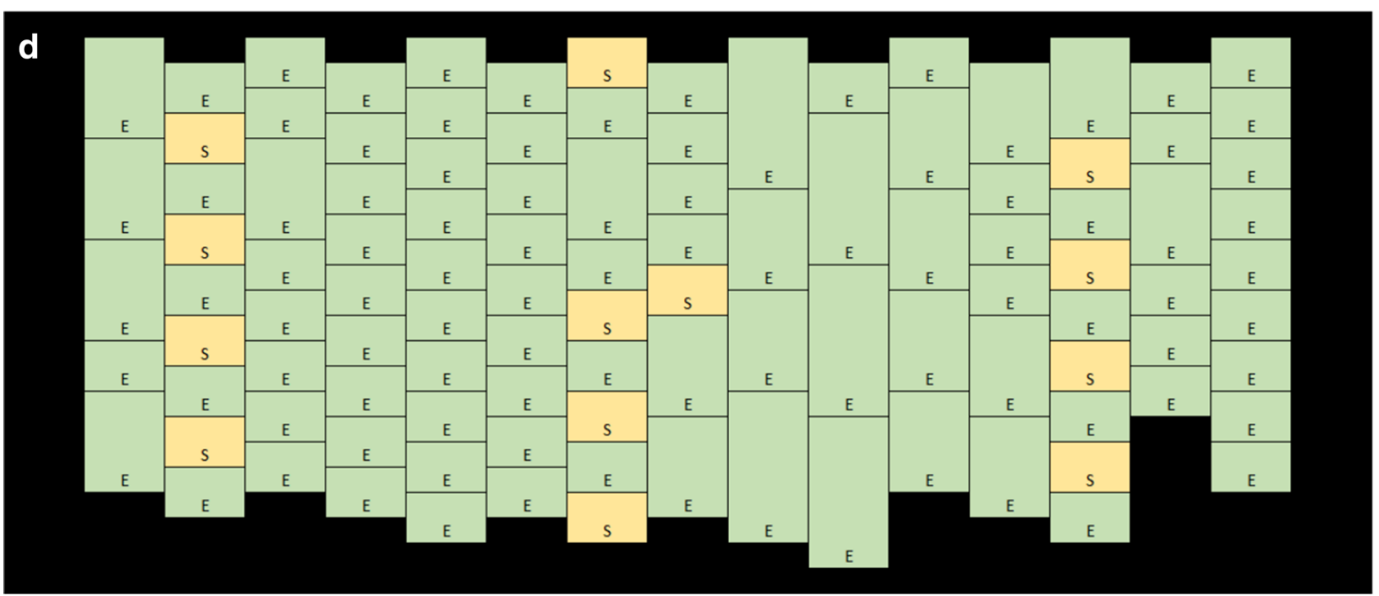

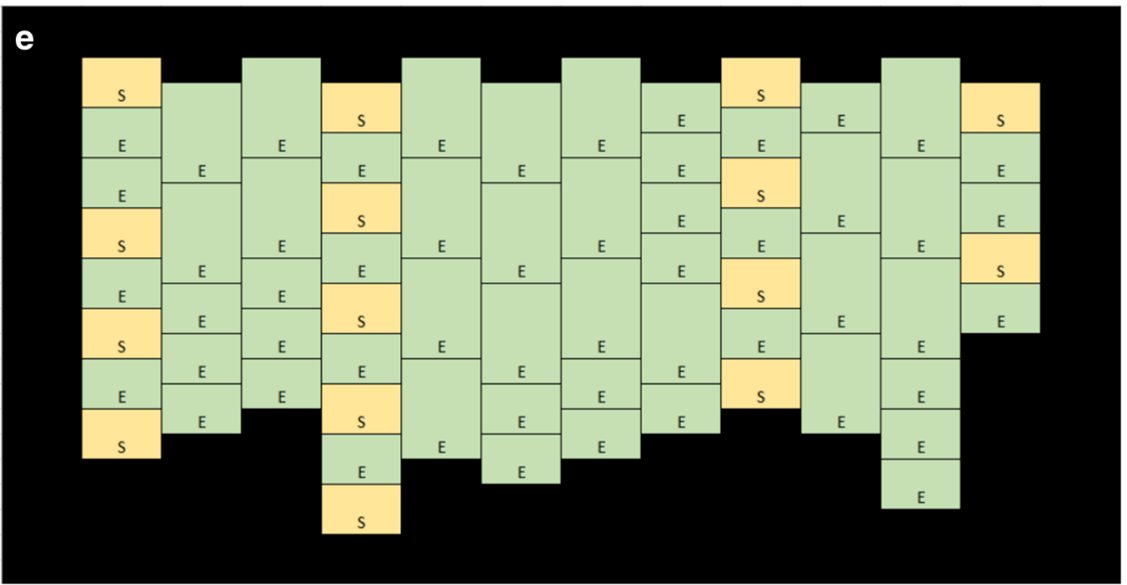

Legend
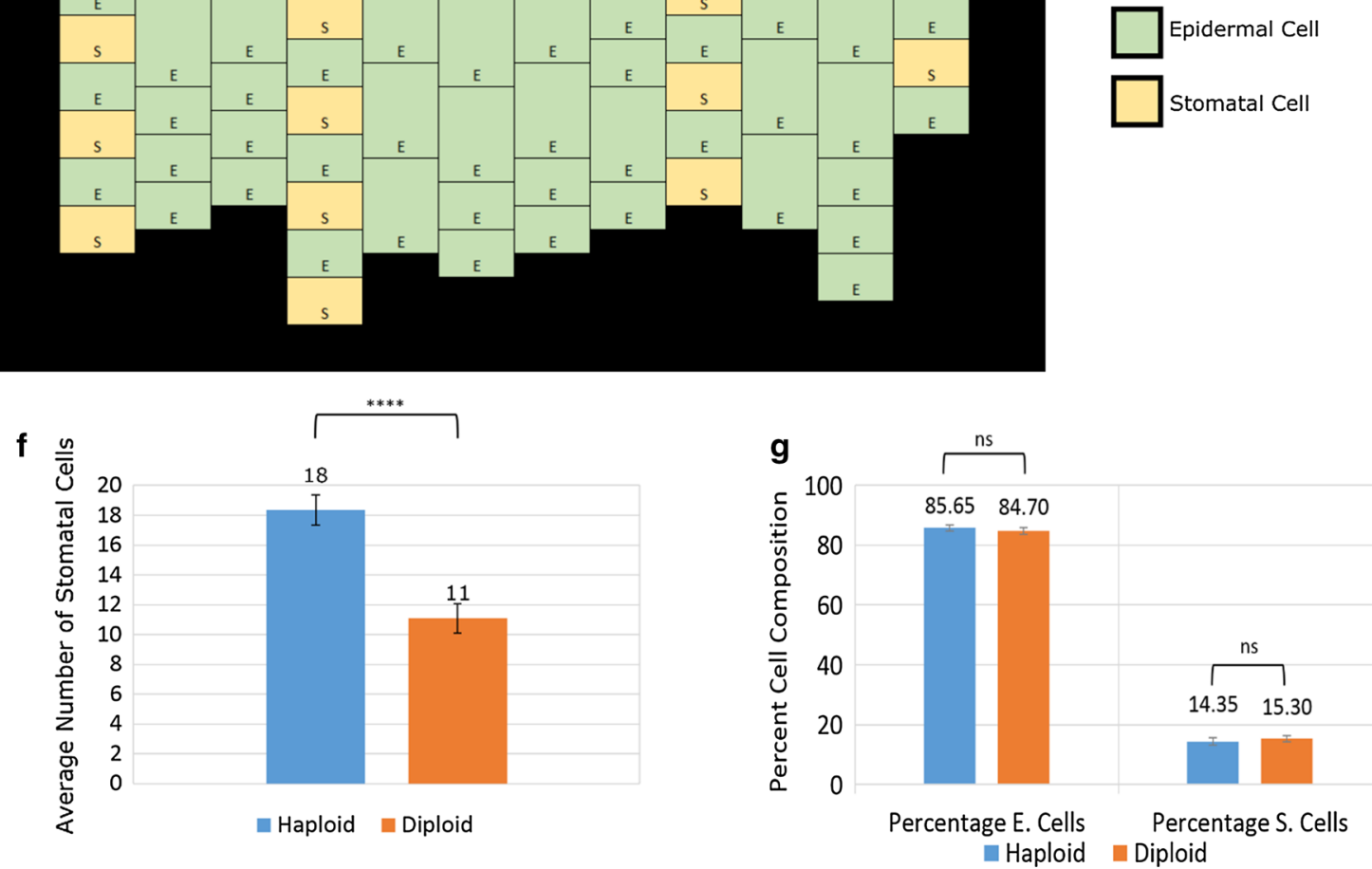
४Fig. 4 Investigation of differences in tissue patterning between haploid and diploid abaxial epidermal surfaces via light microscopy. Representative images of $\mathbf{a}$ haploid and $\mathbf{b}$ diploid interveinal spacing captured using a Nikon Eclipse T2R microscope with a $\times 10$ objective in a $550 \mu \mathrm{m} \times 550 \mu \mathrm{m}$ field of view. c Average interveinal distance calculated from entire sample set of the original 20 haploid and 20 diploid leaf disks. Diagrams representing $\mathbf{d}$ haploid and $\mathbf{e}$ diploid placement of pavement cells and stomata, used to calculate cell counts and percent composition for abaxial epidermal surfaces. f Average number of stomatal cells per area of leaf disk. The total sum of stomata per leaf disk was calculated for both haploid and diploid sets and then averaged. $\mathbf{g}$ Total percent cell composition, broken down into percentage of epidermal cells and percentage of stomata cells per leaf area, for haploid and diploid leaf disk samples

qPCR analysis, where there is no step involved that could allow for DNA loss. Comparatively, when steps are taken to purify DNA samples, there is opportunity for a loss of DNA content at various rates.

All chemical combinations and the procedures for the DNA extraction were optimized for maximum DNA recovery and verified with various plant tissue materials. A repeated DNA extraction from leftover leaf tissue debris showed that only a few more percent more DNA was recovered compared to the initial round (data not shown). The results suggested that DNA yields were quite high and very repeatable. The relatively small standard deviations suggested that the DNA quantification method was reliable. The preliminary conclusion based on these results is that there was essentially no difference in DNA amount per leaf disk between haploid and diploid plants. Instead, our findings suggest that the number of cells are different per unit of leaf area between different ploidy leaf tissues which results in similar levels of DNA content.

Examination of the leaf cell morphologies in those leaves indicated that the haploid plants pack significantly more cells into the same leaf area: epidermal pavement cells in particular are much smaller in haploids than in diploids. The mesophyll cells are also smaller in the interveinal space, although those underneath the minor veins are equivalently sized. Interestingly, in the diploid plants, the mesophyll cells under the minor veins were quite small compared to those between the veins. This raises the question of whether the correlation between ploidy level and size is more pronounced for larger cells programmed to undergo a significant expansion in cell volume. This is consistent to what was found in the sepal epidermis of Arabidopsis, which can accommodate extremely large cells that are octoploid, 16- or 32-ploid (Roeder et al. 2010). We found that the guard cells in haploids were noticeably smaller than in diploids, but stomata patterning within the tissue, and the ratio of guard cells to pavement cells did not change. This indicates that the core epidermal patterning pathways are unaffected by the ploidy content of the composite cells.
Due to limits of the confocal microscope and cell clearing techniques, the adaxial epidermis and mesophyll layers within the leaf disk could not clearly be captured in all of the Z-stack images collected for this experiment. In an attempt to obtain a more comprehensive analysis of differences in epidermal and mesophyll content in haploid and diploid plants, five haploid and five diploid leaf disk samples were selected for additional analysis. These ten leaf disk samples were selected based on the clarity of the adaxial epidermal and mesophyll layer in the Z-Stack. The additional data analysis included: mesophyll cell counts outside of the initial $200 \mu \mathrm{m} \times 100 \mu \mathrm{m}$ region beneath the minor leaf vein, a combined mesophyll cell count and mesophyll cell area measurements, and finally, interveinal spacing. Epidermal tissue-level patterning was analyzed with respect to the placement and proportion of stomata to pavement cells. To broaden this experiment in the future, alternative clearing techniques could be used to determine if autofluorescence signals in the leaf tissue can be reduced. This would result in a clearer Z-stack image, able to clearly see the adaxial layers of the leaf disk in all sample images. Another viable solution moving forward would be to use a chloral hydrate to clear auto-fluorescent secondary metabolites from the cell walls.

A significant difference was discovered in the mesophyll cell counts, driven by the smaller size of mesophyll cells in the interveinal space of the haploids. This could suggest that when cells are confined by larger leaf features, such as a minor leaf vein, their growth is limited due to the lack of cellular space to expand. This could also explain why in regions outside of the restrictions of the minor leaf vein, one may see a significant difference between haploid and diploid cell counts, as a result of the additional cellular space available for expansion in diploids. Our study is consistent with prior observations that cell size correlates with ploidy level. Our observations of maize haploids and diploids of the same genotype indicate that the haploids maintain normal cellular patterning in the epidermis and mesophyll, but they pack more cells into the same amount of space as diploid plants. It has been observed in multiple species that mature haploid plants are significantly smaller in size compared to their diploid counterparts; the same is true of their cells.

While the original objective of this study was to use qPCR assay to determine ploidy from leaf tissue punches, epidermal cell size could still be a useful method for distinguishing haploids, diploids, and doubled haploids. One could develop a system for identifying haploids based on epidermal cell size using rapid, microscopic automated screening technology. Additionally, with the difference in leaf morphology and pavement cell size and content, it would be theoretically possible for one to employ multispectral or hyperspectral imaging techniques to aid in the observation of differences visually. 
Author contribution statement DS: experimental design and management, embryo extraction, imaging, ploidy analysis, image analysis, data presentation, manuscript writing and editing; JH: experimental design, embryo extraction, imaging, ploidy analysis, image analysis, data presentation, manuscript writing; YJ: DNA extraction, qPCR analysis; WY: experimental guidance and theory, data display; WL: experimental management, material acquisition and experimental support; TK: experimental design, guidance and theory, imaging support, analysis and interpretation, manuscript editing.

Acknowledgements We thank T. Liebler and J. Roberts for plant care and crossing. Thank you to I. Jepson, L. Shi and P. Drayton for lab space and personnel support.

Open Access This article is licensed under a Creative Commons Attribution 4.0 International License, which permits use, sharing, adaptation, distribution and reproduction in any medium or format, as long as you give appropriate credit to the original author(s) and the source, provide a link to the Creative Commons licence, and indicate if changes were made. The images or other third party material in this article are included in the article's Creative Commons licence, unless indicated otherwise in a credit line to the material. If material is not included in the article's Creative Commons licence and your intended use is not permitted by statutory regulation or exceeds the permitted use, you will need to obtain permission directly from the copyright holder. To view a copy of this licence, visit http://creativecommons.org/licenses/by/4.0/.

\section{References}

Abendroth LJ, Elmore RW, Boyer MJ, Marlay SK (2011) Corn growth and development. PMR 1009. Iowa State University Extension, Ames, Iowa

Chaikam V, Nair SK, Babu R, Martinez L, Tejomurtula J, Boddupalli PM (2015) Analysis of effectiveness of R1-nj anthocyanin marker for in vivo haploid identification in maize and molecular markers for predicting the inhibition of R1-nj expression. Theor Appl Genet 128(1):159-171

Chang MT, Coe E (2009) Molecular genetics approaches to maize improvement. Springer, Berlin, pp 127-142

Choe E, Carbonero CH, Mulvaney K, Rayburn AL, Mumm RH (2012) Improving in vivo maize doubled haploid production efficiency through early detection of false positives. Plant Breed 131(3):399-401

Deepak SA, Kottapalli KR, Rakwal R, Oros G, Rangappa KS, Iwahashi H, Masuo Y, Agrawal GK (2007) Real-time PCR: revolutionizing detection and expression analysis of genes. Curr Genom $8: 234-251$
Ji Y (2018) Methods and compositions for preserving tissues and nucleic acids. WO 2018/169754A1

Kelliher T, Starr D, Richbourg L, Chintamanani S, Delzer B, Nuccio ML, Green J, Chen Z, McCuiston J, Wang W, Liebler T, Bullock P, Martin B (2017) MATRILINEAL, a sperm-specific phospholipase, triggers maize haploid induction. Nature 542:105-109

Kurihara D, Mizuta Y, Sato Y, Higashiyama T (2015) ClearSee: a rapid optical clearing reagent for whole-plant fluorescence imaging. Development 142:4168-4179

Larcher S, Ermolli M, Querci M, Mazzara M, Cordeil S, Van den Eede G (2008) Event-specific method for the quantification of maize line Bt11 using real-time PCR. In: Joint Research Centre Institute for Health and Consumer Protection. JRC48909. https://doi. org/10.2788/4370

MEKU Erich Pollähne GmbH (2019) http://www.meku-pollaehne.de/ Home/home.html

Molenaar WS, Couto EGO, Piepho HP, Melchinger AE (2019) Early diagnosis of ploidy status in doubled haploid production of maize by stomata length and flow cytometry measurements. Plant Breed 138(2):1-11

Nakayama Y, Yamaguchi H, Einaga N, Esumi M (2016) Pitfalls of DNA quantification using DNA-binding fluorescent dyes and suggested solutions. PLoS One 11(3):e0150528

Nanda DK, Chase SS (1966) An embryo marker for detecting monoploids of maize (Zea mays L.). Crop Sci 6(2):213-215

Ravi M, Chan SWL (2010) Haploid plants produced by centromeremediated genome elimination. Nature 464(7288):615-618

Roeder AHK, Chickarmane V, Cunha A, Obara B, Manjunath BS, Meyerowitz EM (2010) Variability in the control of cell division underlies sepal epidermal patterning in Arabidopsis thaliana. PLoS Biol 8(5):e1000367

Roeder AHK, Cunha A, Ohno CK, Meyerowitz EM (2012) Cell cycle regulates cell type in the Arabidopsis sepal. Development 139:4416-4427

Scholdberg TA, Norden TD, Nelson DD, Jenkins GR (2009) Evaluating precision and accuracy when quantifying different endogenous control reference genes in maize using real-time PCR. J Agric Food Chem 57:2903-2911

Sugimoto-Shirasu K, Roberts K (2003) "Big it up": endoreduplication and cell-size control in plants. Curr Opin Plant Biol 6:544-553

Suni MA, Dunn HS, Orr PL, de Laat R, Sinclair E, Ghanekar SA, Bredt BM, Dunne JF, Maino VC, Maecker HT (2003) Performance of plate-based cytokine flow cytometry with automated data analysis. BMC Immunol 4:9

Xin Z, Chen J (2012) A high throughput DNA extraction method with high yield and quality. Plant Methods 8:26-32

Yao L, Zhang Y, Liu C, Liu Y, Wang Y, Liang D, Liu J, Sahoo G, Kelliher T (2018) OsMATL mutation induces haploid seed formation in indica rice. Nat Plants 4(8):530-533

Publisher's Note Springer Nature remains neutral with regard to jurisdictional claims in published maps and institutional affiliations. 